\title{
Penicillin allergy
}

\author{
David J. McCullagh MB BCh BAO, Derek K. Chu MD PhD
}

Cite as: CMAJ 2019 February 25;191:E231. doi: 10.1503/cmaj.181117

CMAJ Podcasts: author interview at https://soundcloud.com/cmajpodcasts/181117-five

1 p

Penicillin allergy is commonly reported, but 9 out of 10 times, penicillin will be tolerated if administered

About $10 \%$ of people report a penicillin allergy, but $90 \%-95 \%$ of those individuals are not truly allergic. ${ }^{1}$ Reasons for this include mislabelling intolerances as allergies and waning of immunoglobulin E-mediated allergy over time.,3

\section{The label of penicillin allergy is bad for patients and the health care system}

More than just a problem for prescribers of antibiotics, the label of penicillin allergy is associated with the use of costly and less effective secondline and broad-spectrum antibiotics, ${ }^{1-3}$ a $55 \%$ increased risk of acquiring methicillin-resistant Staphylococcus aureus, and a 35\% increased risk of Clostridium difficile infection (Appendix 1, available at www.cmaj.ca/ lookup/suppl/doi:10.1503/cmaj.181117/-/DC1). ${ }^{4}$

Patients reporting penicillin allergy can be easily risk stratified to determine whether they require specialist evaluation

A known adverse effect of a penicillin (e.g., nausea) should not be documented as an allergy; likewise, avoidance of penicillin is unnecessary in those with a family history of penicillin allergy without a personal history of one, or in those who have since tolerated penicillin (low-risk). In those who have experienced severe delayed hypersensitivity reactions such as drug reaction with eosinophilia and systemic symptoms or StevensJohnson syndrome and toxic epidermal necrolysis (high-risk), $\beta$-lactams should be strictly avoided; allergy skin testing is contraindicated. Patients reporting probable or unclear immunoglobulin $\mathrm{E}$ reactions that may present as rapid-onset urticaria, angioedema or anaphylaxis (intermediate risk) should be referred (Appendix 1).2,3

Penicillin allergy is lost over time, with resolution in $80 \%$ of people over 10 years, and in $50 \%$ over 5 years

Those with remote (>10 yr) reactions are unlikely to still be allergic and should be tested before challenge with penicillin. ${ }^{1-3}$ If there is an acute indication for antibiotics, expert consultation is preferable to guide testing versus empiric therapy.

5 Allergy referral and testing is vastly underused, but is safe, accurate, rapid and cost-effective

Allergy testing over 1-2 hours using a combination of skin and challenge testing by trained personnel has been shown to be safe and effective for children, adults, inpatients and outpatients, with a negative predictive value close to $100 \% .^{1-3}$

\section{References}

1. Sacco KA, Bates A, Brigham TJ, et al. Clinical outcomes following inpatient penicillin allergy testing: a systematic review and meta-analysis. Allergy 2017;72:1288-96.

2. Shenoy ES, Macy E, Rowe T, et al. Evaluation and management of penicillin allergy: a review. JAMA 2019;321:188-99.

3. Joint Task Force on Practice Parameters; American Academy of Allergy, Asthma and Immunology; American College of Allergy, Asthma and Immunology; Joint Council of Allergy, Asthma and Immunology. Drug allergy: an updated practice parameter. Ann Allergy Asthma Immunol 2010;105:259-73.

4. Blumenthal KG, Lu N, Zhang Y, et al. Risk of meticillin resistant Staphylococcus aureus and Clostridium difficile in patients with a documented penicillin allergy: population based matched cohort study. BMJ 2018;361:k2400.

\section{Competing interests: None declared.}

This article has been peer reviewed.

Affiliations: Divisions of Infectious Disease (McCullagh), and Clinical Immunology and Allergy (Chu), Department of Medicine, McMaster University, Hamilton, Ont.

Correspondence to: David McCullagh, david.mccullagh@medportal.ca; or Derek Chu, chudk@mcmaster.ca

CMAJ invites submissions to "Five things to know about ..." Submit manuscripts online at http://mc. manuscriptcentral.com/cmaj 